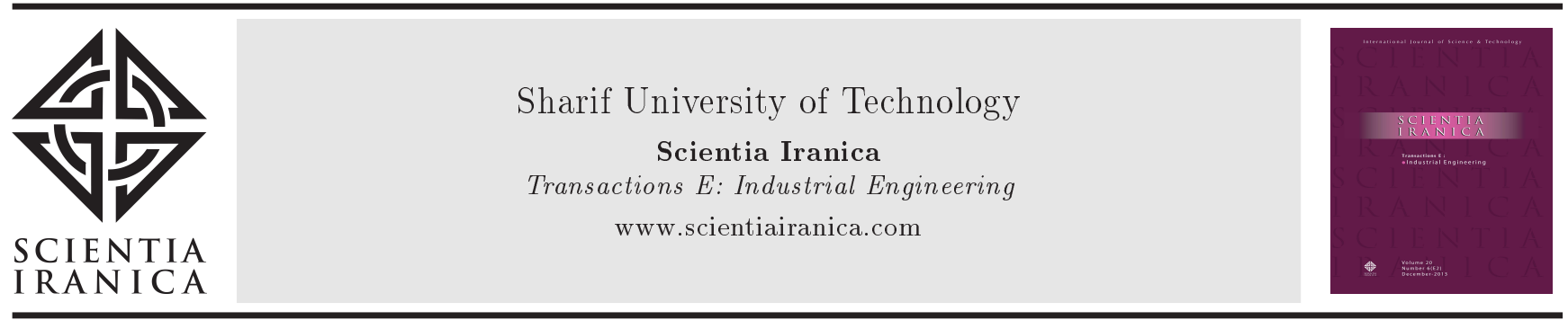

\title{
Managing interval-valued multiplicative hesitant fuzzy information in GDM problems
}

\author{
D. $\mathrm{Yu}^{\mathrm{a}, \mathrm{b}, *}$ and D.-F. Li ${ }^{\mathrm{a}}$ \\ a. School of Economics and Management, Fuzhou University, Fuzhou, Fujian 350108, China. \\ b. School of Information, Zhejiang University of Finance and Economics, Hangzhou, Zhejiang 310018, China. \\ Received 7 September 2014; received in revised form 20 December 2014; accepted 1 August 2015
}

\author{
KEYWORDS \\ Hesitant fuzzy set; \\ Interval-valued \\ multiplicative hesitant \\ fuzzy set; \\ Aggregation operator; \\ GDM; \\ college application.
}

\begin{abstract}
Inspired by the idea of multiplicative preference relation as well as intervalvalued hesitant fuzzy set, we introduce a new preference relation called interval-valued multiplicative hesitant fuzzy preference structure. It is a powerful technique to describe the preference information assessed by different decision makers. The predominant feature is that it allows decision makers to use the interval-valued hesitant fuzzy information in describing the preference structure. We focus on the information aggregation methods for interval-valued multiplicative hesitant fuzzy preference information and propose a series of useful aggregation operators. Moreover, we propose a Group Decision-Making (GDM) method based on the proposed aggregation operators under interval-valued multiplicative hesitant fuzzy environment. A real case about college application problem is presented to show usefulness and effectiveness of our proposed method.
\end{abstract}

(C) 2016 Sharif University of Technology. All rights reserved.

\section{Introduction}

Decision analysis is a regular activity encountered in our daily life and it refers to selection of the most desirable alternatives from a set of possible schemes by using some quantitative methods [1-3]. Multiplicative and additive preference relations are two main techniques to capture the preference structure of the decision makers when they compare alternatives with each other [1,4-6].

Multiplicative preference relations are based on the Saaty's 1-9 scale and have been investigated by many scholars. Chiclana et al. [7] focused on the aggregation methods of the multiplicative fuzzy preference information in Group Decision-Making (GDM) problems. Based on the C-OWA operator [8], Xu [9] extended the fuzzy preference to interval-valued fuzzy environment and introduced an effective method for

*. Corresponding author. Tel.: +8615336884185; Fax: +865718755713

E-mail address: yudejian62@126.com (D. Yu);

lidengfeng@fzu.edu.cn (D.-F.Li) the GDM problem. Fan et al. [10] focused on the GDM problem, where the decision information is expressed by multiplicative fuzzy preference relations, and proposed a fuzzy programming model. Genç et al. [11] extended the fuzzy preference orderings and studied the consistent problem of the interval fuzzy preference relation. Xia et al. [12] extended the multiplicative fuzzy preference relations to intuitionistic fuzzy environment and proposed the intuitionistic multiplicative preference relations. Based on Xia et al. [12], Jiang et al. [13] studied the consistency problem and proposed a series of useful compatibility measures and consensus models for intuitionistic multiplicative GDM problems. Xia and Xu [14] introduced the generalized operations for intuitionistic multiplicative preference information based on Archimedes rules and then introduced some generalized aggregation operators. For capturing the interrelations between the aggregated arguments, the power average and Choquet Integral were used for the aggregation operators. Some other research results about intuitionistic multiplicative preference information are shown in Jiang and 
$\mathrm{Xu}$ [15] and $\mathrm{Yu}$ and Fang [16]. Yu and $\mathrm{Xu}$ [17] introduced the concept of intuitionistic multiplicative triangular fuzzy set and developed a series of aggregation operators for multi-attribute decision making.

Multiplicative Hesitant Fuzzy Set (MHFS) has been introduced by Xia [18] based on the multiplicative preferences relations. The main difference between the MHFS and Hesitant Fuzzy Set (HFS) [19-21] is the difference in adopted preference information. MHFS is complementary to HFS. Recently, MHFS has attracted attention of many researchers. Xia and $\mathrm{Xu}[22]$ proposed a series of multiplicative hesitant fuzzy information aggregation operators using algebraic operational laws, based on which a new GDM method is studied. Unlike the aggregation operators proposed by Xia and Xu [22], Yu [23] proposed a group of Einstein operations based operators for the multiplicative hesitant fuzzy information. Wang et al. [24] focused on the compatibility measures and aggregation operators combined with the power mean. Liao et al. [25] investigated the problem of consistency of the multiplicative hesitant fuzzy decision-making matrix. Some concepts such as multiplicative consistency, acceptable multiplicative consistency, and perfect multiplicative consistency are introduced.

It should be noted that the membership degree of the MHFS is expressed by a determined value between $\frac{1}{9}$ and 9 . However, in many practical situations, it is hard to use the crisp numbers to express the membership degree and the interval number is a very good choice. In this regard, it will be more applicable to practical problems if we use the interval numbers that belong to $\left[\frac{1}{9}, 9\right]$ instead of determined numbers to express the membership degree of the MHFS. Therefore, in this paper, we first propose an extended MHFS, called Interval MHFS (IMHFS), and then study its applications.

The next section gives some basic concepts and then introduces the IMHFS. Section 3 proposes a series of aggregation operators based on three different scenarios. Section 4 proposes a new GDM method using Interval Multiplicative Hesitant Fuzzy Numbers (IMHFNs) and an actual application case about voluntary choice of college entrance examination in China is presented. Section 5 concludes this paper and the future directions in IMHFS are also outlined.

\section{MHFS and IMHFS}

MHFS was proposed by Xia [18] and its structure is very similar to that of HFS. However, MHFS is based on 1-9 ratio scale while the HFS takes advantage of the 0.1-0.9 scale. The definition of MHFS is given as follows [18].
Definition 1. Suppose there is an objective set and marked as $X$; the MHFS is defined as:

$$
D=\left\{\left\langle x, \rho_{D}(x)\right\rangle \mid x \in X\right\} .
$$

In Eq. (1), the function $\rho_{D}(x)$ is valued in the interval $[1 / 9,9]$.

As the basic component of MHFS, Multiplicative Hesitant Fuzzy Number (MHFN) was marked as $\rho=$ $\rho(x)$, which was also defined by Xia [18].

Based on Definition 1, in the following, we give the definition of IMHFS.

Definition 2. Suppose there is an objective set and marked as $X$; the IMHFS is defined as:

$$
D=\left\{\left\langle x, \tilde{\rho}_{D}(x)\right\rangle \mid x \in X\right\},
$$

where $\tilde{\rho}_{D}(x)=\left[\tilde{\rho}_{D}^{-}(x), \tilde{\rho}_{D}^{+}(x)\right]$ is the membership degree interval with the conditions that $\tilde{\rho}_{D}(x) \in\left[\frac{1}{9}, 9\right]$.

Inspired by the idea of MHFN, we define $\tilde{\rho}_{D}(x)$ as the IMHFN. For any IMHFN $\tilde{\rho}_{D}(x)$, the score is defined as:

$$
s(\tilde{\rho})=\sqrt[1 / \Delta g]{\prod_{\eta \in g} \eta^{+} \cdot \eta^{-}}
$$

$\left[\eta^{-}, \eta^{+}\right]$is an interval number among the possible intervals in $\tilde{\rho}_{D}(x)$. For any given two IMHFNs $\tilde{\rho}_{1}$ and $\tilde{\rho}_{2}$, if $s\left(\tilde{\rho}_{1}\right)>s\left(\tilde{\rho}_{2}\right)$, then $\tilde{\rho}_{1} \succ \tilde{\rho}_{2}$.

\section{Aggregation operators for IMHFNs}

The information aggregation is essential to many fields. In this section, some series of new types of operators, called IMHF aggregation operators, are proposed. Three different operational laws, i.e. algebraic operational laws, Einstein operational laws, and Archimedes operational laws, are adopted.

\subsection{Operation laws for IMHFNs}

In our previous work [26], some algebraic operational laws and corresponding aggregation operators for IMHF information have been investigated, based on which we introduce some IMHF information operational laws.

Definition 3. Suppose $\tilde{\rho}_{1}$ and $\tilde{\rho}_{2}$ to be two IMHFNs and $\lambda$ be a real number greater than zero; then, the algebraic operational laws of the IMHF information are defined as follows:

(1) $\tilde{\rho}_{1}^{\lambda}=\cup_{\tilde{\eta}_{1} \in \tilde{\rho}_{1}}$

$$
\left\{\left[\frac{\eta_{1}^{-\lambda}}{\left(1+\eta_{1}^{-}\right)^{\lambda}-\eta_{1}^{-\lambda}}, \frac{\eta_{1}^{+\lambda}}{\left(1+\eta_{1}^{+}\right)^{\lambda}-\eta_{1}^{+\lambda}}\right]\right\},
$$


(2) $\lambda \tilde{\rho}_{1}=\cup_{\tilde{\eta}_{1} \in \tilde{\rho}_{1}}\left\{\left[\left(1+\eta_{1}^{-}\right)^{\lambda}-1,\left(1+\eta_{1}^{+}\right)^{\lambda}-1\right]\right\}$,

(3) $\quad \tilde{\rho}_{1} \oplus \tilde{\rho}_{2}=\cup_{\tilde{\eta}_{1} \in \tilde{\rho}_{1}, \tilde{\eta}_{2} \in \tilde{\rho}_{2}}$

$$
\left\{\left[\eta_{1}^{-}+\eta_{2}^{-}+\eta_{1}^{-} \eta_{2}^{-}, \eta_{1}^{+}+\eta_{2}^{+}+\eta_{1}^{+} \eta_{2}^{+}\right]\right\},
$$

(4) $\tilde{\rho}_{1} \otimes \tilde{\rho}_{2}=\cup_{\tilde{\eta}_{1} \in \tilde{\rho}_{1}, \tilde{\eta}_{2} \in \tilde{\rho}_{2}}$

$$
\left(\left[\frac{\eta_{1}^{-} \eta_{2}^{-}}{\eta_{1}^{-}+\eta_{2}^{-}+1}, \frac{\eta_{1}^{+} \eta_{2}^{+}}{\eta_{1}^{+}+\eta_{2}^{+}+1}\right]\right)
$$

Based on the Einstein operations, Xia et al. [12] introduced some operational laws for multiplicative intuitionistic fuzzy information, inspired by which we propose some Einstein operational laws for IMHF information.

Definition 4. Suppose $\tilde{\rho}_{1}$ and $\tilde{\rho}_{2}$ to be two IMHFNs and $\lambda$ be a real number greater than zero; then, the Einstein operational laws of the IMHF information are defined as follows:

(5) $\quad \tilde{\rho}_{1}^{\lambda}=\cup_{\tilde{\eta}_{1} \in \tilde{\rho}_{1}}$

$$
\left\{\left[\frac{2 \eta_{1}^{-\lambda}}{\left(2+\eta_{1}^{-}\right)^{\lambda}-\eta_{1}^{-\lambda}} \frac{2 \eta_{1}^{+\lambda}}{\left(2+\eta_{1}^{+}\right)^{\lambda}-\eta_{1}^{+\lambda}}\right]\right\},
$$

(6) $\lambda \tilde{\rho}_{1}=\cup_{\tilde{\eta}_{1} \in \tilde{\rho}_{1}}$

$$
\left\{\left[\frac{\left(1+2 \eta_{1}^{-}\right)^{\lambda}-1}{2}, \frac{\left(1+2 \eta_{1}^{+}\right)^{\lambda}-1}{2}\right]\right\}
$$

(7) $\quad \tilde{\rho}_{1} \oplus \tilde{\rho}_{2}=\cup_{\tilde{\eta}_{1} \in \tilde{\rho}_{1}, \tilde{\eta}_{2} \in \tilde{\rho}_{2}}$

$$
\begin{aligned}
\left\{\left[\frac{\left(1+2 \eta_{1}^{-}\right)\left(1+2 \eta_{2}^{-}\right)-1}{2},\right.\right. \\
\left.\left.\quad \frac{\left(1+2 \eta_{1}^{+}\right)\left(1+2 \eta_{2}^{+}\right)-1}{2}\right]\right\},
\end{aligned}
$$

(8) $\quad \tilde{\rho}_{1} \otimes \tilde{\rho}_{2}=\cup_{\tilde{\eta}_{1} \in \tilde{\rho}_{1}, \tilde{\eta}_{2} \in \tilde{\rho}_{2}}$

$$
\begin{gathered}
\left(\left[\frac{2 \eta_{1}^{-} \eta_{2}^{-}}{\left(2+\eta_{1}^{-}\right)\left(2+\eta_{2}^{-}\right)-\eta_{1}^{-} \eta_{2}^{-}},\right.\right. \\
\left.\left.\quad \frac{2 \eta_{1}^{+} \eta_{2}^{+}}{\left(2+\eta_{1}^{+}\right)\left(2+\eta_{2}^{+}\right)-\eta_{1}^{+} \eta_{2}^{+}}\right]\right) .
\end{gathered}
$$

Definition 3 is based on algebraic operational laws while Definition 4 is based on Einstein operational laws. In the following, we generalize the two kinds of operational laws above through the introduction of the Archimedean operations.
Definition 5. Suppose $\tilde{\rho}_{1}$ and $\tilde{\rho}_{2}$ to be two IMHFNs and $\lambda$ be a real number greater than zero; then, Archimedean operational laws of the IMHF information are defined as follows:

(9) $\quad \tilde{\rho}_{1}^{\lambda}=\cup_{\tilde{\eta}_{1} \in \tilde{\rho}_{1}}$

$$
\left\{\left[g^{-1}\left(g\left(h\left(\eta_{1}^{-}\right)\right)^{\lambda}\right), g^{-1}\left(\left(g\left(\eta_{1}^{+}\right)\right)^{\lambda}\right)\right]\right\},
$$

(10) $\lambda \tilde{\rho}_{1}=\cup_{\tilde{\eta}_{1} \in \tilde{\rho}_{1}}$

$$
\left\{\left[h^{-1}\left(\left(h\left(\eta_{1}^{-}\right)\right)^{\lambda}\right), h^{-1}\left(\left(h\left(\eta_{1}^{+}\right)\right)^{\lambda}\right)\right]\right\},
$$

(11) $\quad \tilde{\rho}_{1} \oplus \tilde{\rho}_{2}=\cup_{\tilde{\eta}_{1} \in \tilde{\rho}_{1}, \tilde{\eta}_{2} \in \tilde{\rho}_{2}}$

$$
\left\{\left[h^{-1}\left(h\left(\eta_{1}^{-}\right) \cdot h\left(\eta_{2}^{-}\right)\right), h^{-1}\left(h\left(\eta_{1}^{+}\right) \cdot h\left(\eta_{2}^{+}\right)\right)\right]\right\},
$$

(12) $\quad \tilde{\rho}_{1} \otimes \tilde{\rho}_{2}=\cup_{\tilde{\eta}_{1} \in \tilde{\rho}_{1}, \tilde{\eta}_{2} \in \tilde{\rho}_{2}}$

$$
\left(\left[\left[g^{-1}\left(g\left(\eta_{1}^{-}\right) \cdot g\left(\eta_{2}^{-}\right)\right), g^{-1}\left(g\left(\eta_{1}^{+}\right) \cdot g\left(\eta_{2}^{+}\right)\right)\right]\right]\right) .
$$

In Definition 5, $g$ represents a strictly decreasing function and has a very close relationship with $h$, satisfying $h(t)=g\left(\frac{1}{t}\right)$. The function $g$ is a generalized form and when it takes some common forms, (9)-(12) would be transformed.

\subsection{Some aggregation operators for IMHFNs}

Definition 6. Suppose $\tilde{\rho}_{i}(i=1,2, \cdots, n)$ to be a collection of IMHFNs and $w_{i}$ be the weight of $\tilde{\rho}_{i}$, $w_{i} \in[0,1]$ and $\sum_{i=1}^{n} w_{i}=1$. Then, the IMHF Weighted Average operator (IMHFWA) and IMHF Weighted Heometric operator (IMHFWG) are defined as follows:

\section{IMHFWA:}

$$
\left(\tilde{\rho}_{1}, \tilde{\rho}_{2}, \cdots, \tilde{\rho}_{n}\right)=w_{1} \tilde{\rho}_{1} \oplus w_{2} \tilde{\rho}_{2} \oplus \cdots \oplus w_{n} \tilde{\rho}_{n},
$$

\section{IMHFWG:}

$$
\left(\tilde{\rho}_{1}, \tilde{\rho}_{2}, \cdots, \tilde{\rho}_{n}\right)=\tilde{\rho}_{1}^{w_{1}} \otimes \tilde{\rho}_{2}^{w_{2}} \otimes \cdots \otimes \tilde{\rho}_{n}^{w_{n}} .
$$

Combined with the well-known OWA operator (Yager, 1988), in the following, we introduce the IMHFOWA and IMHFOWG operators.

Definition 7. Suppose $\tilde{\rho}_{i}(i=1,2, \cdots, n)$ to be a collection of IMHFNs; the IMHFOWA and IMHFOWG operators are defined as follows:

\section{IMHFOWA:}

$$
\left(\tilde{\rho}_{1}, \tilde{\rho}_{2}, \cdots, \tilde{\rho}_{n}\right)=\omega_{1} \tilde{\rho}_{\sigma(1)} \oplus \omega_{2} \tilde{\rho}_{\sigma(2)} \oplus \cdots \oplus \omega_{n} \tilde{\rho}_{\sigma(n)},
$$

IMHFOWG:

$$
\left(\tilde{\rho}_{1}, \tilde{\rho}_{2}, \cdots, \tilde{\rho}_{n}\right)=\tilde{\rho}_{\sigma(1)}^{\omega_{1}} \otimes \tilde{\rho}_{\sigma(2)}^{\omega_{2}} \otimes \cdots \otimes \tilde{\rho}_{\sigma(n)}^{\omega_{n}},
$$


where $\left(\omega_{1}, \omega_{2}, \cdots, \omega_{n}\right)$ is the associate weight vector of $\tilde{\rho}_{i}(i=1,2, \cdots, n)$.

In order to apply the IMHFOWA and IMHFOWG operators to the decision-making area, we should further research these two operators and transform them to some more simple forms. Based on the algebraic operational laws of IMHF information defined in Definition 3, the following useful results can be obtained.

Theorem 1. Suppose $\tilde{\rho}_{i}(i=1,2, \cdots, n)$ to be a collection of IMHFNs and $w_{i}$ be the weight of $\tilde{\rho}_{i}$, $w_{i} \in[0,1]$, and $\sum_{i=1}^{n} w_{i}=1$. Then:

IMHFWA:

$$
\begin{aligned}
\left(\tilde{\rho}_{1}, \tilde{\rho}_{2}, \cdots, \tilde{\rho}_{n}\right)=\cup_{\tilde{\eta}_{i} \in \tilde{\rho}_{i}}\{ & {\left[\prod_{i=1}^{n}\left(\eta_{i}^{-}+1\right)^{w_{i}}-1,\right.} \\
& \left.\left.\prod_{i=1}^{n}\left(\eta_{i}^{+}+1\right)^{w_{i}}-1\right]\right\},
\end{aligned}
$$

IMHFWG:

$$
\left(\tilde{\rho}_{1}, \tilde{\rho}_{2}, \cdots, \tilde{\rho}_{n}\right)
$$

$$
\begin{array}{r}
=\cup_{\tilde{\eta}_{i}} \in \tilde{\rho}_{i}\left\{\left[\frac{\prod_{i=1}^{n} \eta_{i}^{-w_{i}}}{\prod_{i=1}^{n}\left(1+\eta_{i}^{-}\right)^{w_{i}}-\prod_{i=1}^{n} \eta_{i}^{-w_{i}}},\right.\right. \\
\left.\left.\frac{\prod_{i=1}^{n} \eta_{i}^{+w_{i}}}{\prod_{i=1}^{n}\left(1+\eta_{i}^{+}\right)^{w_{i}}-\prod_{i=1}^{n} \eta_{i}^{+w_{i}}}\right]\right\},
\end{array}
$$

IMHFOWA:

$$
\begin{aligned}
\left(\tilde{\rho}_{1}, \tilde{\rho}_{2}, \cdots, \tilde{\rho}_{n}\right)=\cup_{\tilde{\eta}_{i} \in \tilde{\rho}_{i}}\{ & {\left[\prod_{i=1}^{n}\left(\eta_{\sigma(i)}^{-}+1\right)^{\omega_{i}}-1,\right.} \\
& \left.\left.\prod_{i=1}^{n}\left(\eta_{\sigma(i)}^{+}+1\right)^{\omega_{i}}-1\right]\right\},
\end{aligned}
$$

IMHFOWG:

$$
\begin{aligned}
& \left(\tilde{\rho}_{1}, \tilde{\rho}_{2}, \cdots, \tilde{\rho}_{n}\right) \\
& =\cup_{\tilde{\eta}_{i} \in \tilde{\rho}_{i}}\left\{\left[\frac{\prod_{i=1}^{n} \eta_{\sigma(i)}^{-\omega_{i}}}{\prod_{i=1}^{n}\left(1+\eta_{\sigma(i)}^{-}\right)^{\omega_{i}}-\prod_{i=1}^{n} \eta_{\sigma(i)}^{-\omega_{i}}}\right.\right. \\
& \left.\left.\frac{\prod_{i=1}^{n} \eta_{\sigma(i)}^{+\omega_{i}}}{\prod_{i=1}^{n}\left(1+\eta_{\sigma(i)}^{+}\right)^{\omega_{i}}-\prod_{i=1}^{n} \eta_{\sigma(i)}^{+\omega_{i}}}\right]\right\}
\end{aligned}
$$

In Eqs. (10) and (11), $\left(\omega_{1}, \omega_{2}, \cdots, \omega_{n}\right)$ is the associate weight vector of $\tilde{\rho}_{i}(i=1,2, \cdots, n)$ and $\eta_{\sigma(i)}$ is the $i$ th largest of $\eta_{i}$.

Example 1. Suppose $\tilde{\rho}_{1}=\{[1 / 6,1 / 5],[2 / 3,3 / 4]\}$, $\tilde{\rho}_{2}=\{[3 / 4,2],[1 / 3,3],[1,2]\}$, and $\tilde{\rho}_{2}=\{[4,5]\}$ to be three IMHFNs; then, based on Theorem 1, the aggregated IMHFNs can be obtained as follows:

1. The results based on IMHFWA are obtained as follows:

$$
\begin{aligned}
\tilde{\rho}= & \operatorname{IMHFWA}\left(\tilde{\rho}_{1}, \tilde{\rho}_{2}, \tilde{\rho}_{3}\right) \\
= & \{[1.1693,1.7850],[0.9813,2.0652], \\
& {[1.2680,1.7850],[1.4432,2.1582], } \\
& {[1.2314,2.4760],[1.5544,2.1582]\} s(\tilde{\rho}) } \\
= & 2.5945 .
\end{aligned}
$$

2. The results based on IMHFWG are obtained as follows:

$$
\begin{aligned}
\tilde{\rho}= & \operatorname{IMHFWG}\left(\tilde{\rho}_{1}, \tilde{\rho}_{2}, \tilde{\rho}_{3}\right) \\
= & \{[0.5770,0.8262],[0.4403,0.8886], \\
& {[0.6265,0.8262],[1.0648,1.6302], } \\
& {[0.7571,1.8139],[1.1876,1.6302]\} s(\tilde{\rho}) } \\
= & 0.8739 .
\end{aligned}
$$

Based on the Einstein operational laws of IMHF information defined in Definition 4, we can get the following useful results.

Theorem 2. Suppose $\tilde{\rho}_{i}(i=1,2, \cdots, n)$ to be a collection of IMHFNs, and $w_{i}$ be the weight of $\tilde{\rho}_{i}$, $w_{i} \in[0,1]$, and $\sum_{i=1}^{n} w_{i}=1$. Then:

IMHFWA:

$$
\begin{aligned}
\left(\tilde{\rho}_{1}, \tilde{\rho}_{2}, \cdots, \tilde{\rho}_{n}\right)=\cup_{\tilde{\eta}_{i} \in \tilde{\rho}_{i}}\{ & {\left[\prod_{i=1}^{n} \frac{\left(1+2 \eta_{i}^{-}\right)^{w_{i}}-1}{2},\right.} \\
& \left.\left.\prod_{i=1}^{n} \frac{\left(1+2 \eta_{i}^{+}\right)^{w_{i}}-1}{2}\right]\right\},
\end{aligned}
$$

IMHFWG:

$$
\begin{aligned}
& \left(\tilde{\rho}_{1}, \tilde{\rho}_{2}, \cdots, \tilde{\rho}_{n}\right) \\
& \quad=\cup_{\tilde{\eta}_{i} \in \tilde{\rho}_{i}}\left\{\left[\frac{2 \prod_{i=1}^{n} \eta_{i}^{-w_{i}}}{\prod_{i=1}^{n}\left(2+\eta_{i}^{-}\right)^{w_{i}}-\prod_{i=1}^{n} \eta_{i}^{-w_{i}}},\right.\right.
\end{aligned}
$$




$$
\left.\left.\frac{2 \prod_{i=1}^{n} \eta_{i}^{+w_{i}}}{\prod_{i=1}^{n}\left(2+\eta_{i}^{+}\right)^{w_{i}}-\prod_{i=1}^{n} \eta_{i}^{+w_{i}}}\right]\right\}
$$

IMHFOWA:

$$
\begin{aligned}
\left(\tilde{\rho}_{1}, \tilde{\rho}_{2}, \cdots, \tilde{\rho}_{n}\right)=\cup_{\tilde{\eta}_{i} \in \tilde{\rho}_{i}} & \left\{\left[\prod_{i=1}^{n} \frac{\left(1+2 \eta_{\sigma(i)}^{-}\right)^{\omega_{i}}-1}{2},\right.\right. \\
& \left.\left.\prod_{i=1}^{n} \frac{\left(1+2 \eta_{\sigma(i)}^{+}\right)^{\omega_{i}}-1}{2}\right]\right\},
\end{aligned}
$$

IMHFOWG:

$$
\begin{aligned}
& \left(\tilde{\rho}_{1}, \tilde{\rho}_{2}, \cdots, \tilde{\rho}_{n}\right) \\
& =\cup_{\tilde{\eta}_{i} \in \tilde{\rho}_{i}}\left\{\left[\frac{2 \prod_{i=1}^{n} \eta_{\sigma(i)}^{-\omega_{i}}}{\prod_{i=1}^{n}\left(2+\eta_{\sigma(i)}^{-}\right)^{\omega_{i}}-\prod_{i=1}^{n} \eta_{\sigma(i)}^{-\omega_{i}}},\right.\right. \\
& \left.\left.\frac{2 \prod_{i=1}^{n} \eta_{\sigma(i)}^{+\omega_{i}}}{\prod_{i=1}^{n}\left(2+\eta_{\sigma(i)}^{+}\right)^{\omega_{i}}-\prod_{i=1}^{n} \eta_{\sigma(i)}^{+\omega_{i}}}\right]\right\}
\end{aligned}
$$

Example 2. Take the three IMHFNs in Example 1; for example, based on Theorem 2, the aggregated IMHFNs can be obtained as follows:

1. The results based on IMHFWA:

$$
\begin{aligned}
\tilde{\rho}= & \operatorname{IMHFWA}\left(\tilde{\rho}_{1}, \tilde{\rho}_{2}, \tilde{\rho}_{3}\right) \\
= & \{[1.0536,1.6272],[0.8572,1.8796], \\
& {[1.1510,1.6272],[1.3722,2.0807], } \\
& {[1.1355,2.3870],[1.4895,2.0807]\} s(\tilde{\rho}) } \\
= & 2.2333 .
\end{aligned}
$$

2. The results based on IMHFWG:

$$
\begin{aligned}
\tilde{\rho}= & \operatorname{IMHFWG}\left(\tilde{\rho}_{1}, \tilde{\rho}_{2}, \tilde{\rho}_{3}\right) \\
= & \{[0.6348,0.9369],[0.4821,1.0258], \\
& {[0.6940,0.9369],[1.1099,1.7044], } \\
& {[0.8077,1.9134],[1.2340,1.7044]\} s(\tilde{\rho}) } \\
= & 1.0274 .
\end{aligned}
$$

Based on Archimedean operational laws of the IMHF information defined in Definition 6, we can get the following useful results.
Theorem 3. Suppose $\tilde{\rho}_{i}(i=1,2, \cdots, n)$ to be a collection of IMHFNs and $w_{i}$ be the weight of $\tilde{\rho}_{i}$, $w_{i} \in[0,1]$, and $\sum_{i=1}^{n} w_{i}=1$. Then:

IMHFWA:

$$
\begin{array}{r}
\left(\tilde{\rho}_{1}, \tilde{\rho}_{2}, \cdots, \tilde{\rho}_{n}\right)=\cup_{\tilde{\eta}_{i} \in \tilde{\rho}_{i}}\left\{\left[h^{-1}\left(\prod_{i=1}^{n}\left(h\left(\eta_{i}^{-}\right)\right)^{w_{i}}\right),\right.\right. \\
\left.\left.h^{-1}\left(\prod_{i=1}^{n}\left(h\left(\eta_{i}^{+}\right)\right)^{w_{i}}\right)\right]\right\},
\end{array}
$$

IMHFWG:

$$
\begin{array}{r}
\left(\tilde{\rho}_{1}, \tilde{\rho}_{2}, \cdots, \tilde{\rho}_{n}\right)=\cup_{\tilde{\eta}_{i} \in \tilde{\rho}_{i}}\left\{\left[g^{-1}\left(\prod_{i=1}^{n}\left(g\left(\eta_{i}^{-}\right)\right)^{w_{i}}\right),\right.\right. \\
\left.\left.g^{-1}\left(\prod_{i=1}^{n}\left(g\left(\eta_{i}^{+}\right)\right)^{w_{i}}\right)\right]\right\},
\end{array}
$$

IMHFOWA:

$$
\begin{array}{r}
\left(\tilde{\rho}_{1}, \tilde{\rho}_{2}, \cdots, \tilde{\rho}_{n}\right)=\cup_{\tilde{\eta}_{i} \in \tilde{\rho}_{i}}\left\{\left[h^{-1}\left(\prod_{i=1}^{n}\left(h\left(\eta_{\sigma(i)}^{-}\right)\right)^{\omega_{i}}\right),\right.\right. \\
\left.\left.h^{-1}\left(\prod_{i=1}^{n}\left(h\left(\eta_{\sigma(i)}^{+}\right)\right)^{\omega_{i}}\right)\right]\right\},
\end{array}
$$

IMHFOWG:

$$
\begin{array}{r}
\left(\tilde{\rho}_{1}, \tilde{\rho}_{2}, \cdots, \tilde{\rho}_{n}\right)=\cup_{\tilde{\eta}_{i} \in \tilde{\rho}_{i}}\left\{\left[g^{-1}\left(\prod_{i=1}^{n}\left(g\left(\eta_{\sigma(i)}^{-}\right)\right)^{\omega_{i}}\right),\right.\right. \\
\left.\left.g^{-1}\left(\prod_{i=1}^{n}\left(g\left(\eta_{\sigma(i)}^{+}\right)\right)^{\omega_{i}}\right)\right]\right\} .
\end{array}
$$

\section{Multi-criteria GDM under interval-valued multiplicative hesitant fuzzy environment}

Multi-criteria GDM is also called multiple-objective decisions with finite scheme and has widely been applied in technology, engineering, mathematics, etc. Because there is a profound theoretical significance and wide practical background in various fields, research on multi-criteria GDM problem has always drawn close attention [27-29]. The essence of multi-criteria decision making is to rank the finite alternatives based on the decision-making information. It is composed of two important parts:

1. Acquisition of decision information;

2. Aggregation of the decision information for every alternative based on the appropriate aggregation methods. 
Suppose there is a group of experts $\left(e_{1}, e_{2}, \cdots, e_{p}\right)$ (decision makers) to evaluate a set of alternatives $\left(x_{1}, x_{2}, \cdots, x_{n}\right)$. During the evaluation process, they compare each alternative with others and the comparison results are expressed by interval-valued multiplicative hesitant fuzzy numbers:

$$
\begin{aligned}
\tilde{\rho}_{i j}^{k} & =\cup_{\tilde{\eta}_{i j}^{k} \in \tilde{\rho}_{i j}^{k}}\left\{\left[\eta_{i j}^{-k}, \eta_{i j}^{+k}\right]\right\} \\
& (i, j=1,2, \cdots, n ; k=1,2, \cdots, p) .
\end{aligned}
$$

The meaning of $\tilde{\rho}_{i j}^{k}$ is explained as: $\left[\eta_{i j}^{-k}, \eta_{i j}^{+k}\right] \subseteq$ $[1 / 9,9]$; where $\left[\eta_{i j}^{-k}, \eta_{i j}^{+k}\right]$ is the degree range provided by the decision maker, $e_{k}$, with the meaning of alternative $x_{i}$ being priority over the alternative $x_{j}$. To find the most appropriate alternative, a decision process is defined:

- Step 1. Obtain the comprehensive performance value of alternative $x_{i}$ provided by expert $e^{k}$. This process should take advantage of the IMHFWA, IMHFWG, IMHFOWA, or IMHFOWAG operator to aggregate $\left(\tilde{\rho}_{1 j}^{k}, \tilde{\rho}_{2 j}^{k}, \cdots, \tilde{\rho}_{n j}^{k}\right)$;

- Step 2. Obtain the comprehensive performance value for alternative $x_{i}$. This process should utilize the operators to aggregate all the performance values provided by expert $e^{k}$ and get the final value for alternatives;

- Step 3. Rank the final performance values for each alternative.

\section{Case analysis: Voluntary choice of college entrance examination in China}

College entrance examination in China is a very important event in one's life and it has a very close relationship with the examinee's development in the future. In the last three decades, due to the economy, population, policy, etc., higher education has played a significant role. As the lack of the higher education resources and the number of candidates have increased, the college entrance examination is faced with more and stronger competition. The factors should be considered by students and their parents in voluntary choice of college entrance examination, including school factors, job factors, education factors, geographic location, occupation development factors, etc. Therefore, college entrance examination is a fuzzy multi-criteria GDM problem.

Generally speaking, voluntary choice of college entrance examination is decided by the examinee $\left(e_{1}\right)$ and his/her father $\left(e_{2}\right)$, and mother $\left(e_{3}\right)$. Meanwhile, we suppose the weight vector of the three decision makers (examinee, his/her father, his/her mother) as $(1 / 3,1 / 3,1 / 3)^{T}$. When facing three universities $\left(x_{1}, x_{2}, x_{3}\right)$, the decision makers compare each univer- sity with others and construct the following intervalvalued multiplicative hesitant fuzzy matrices:

$$
\tilde{D}^{1}
$$$$
=\left(\begin{array}{ccc}
\{[1,1]\} & \left\{\left[\frac{1}{4}, \frac{2}{3}\right]\right\} & \left\{\left[\frac{1}{6}, \frac{1}{3}\right],\left[\frac{2}{5}, \frac{2}{3}\right]\right\} \\
\{[1,2],[2,4]\} & \{[1,1]\} & \left\{\left[\frac{1}{5}, \frac{3}{4}\right]\right\} \\
\left\{\left[\frac{1}{7}, \frac{1}{4}\right],\left[\frac{2}{5}, \frac{3}{4}\right]\right\} & \{[2,4]\} & \{[1,1]\}
\end{array}\right),
$$

$\tilde{D}^{2}$

$$
\begin{gathered}
=\left(\begin{array}{ccc}
\{[1,1]\} & \{[1,2],[3,5]\} & \left\{\left[\frac{1}{4}, \frac{2}{3}\right],\left[\frac{1}{6}, \frac{1}{2}\right]\right\} \\
\left\{\left[\frac{3}{4}, \frac{5}{3}\right]\right\} & \{[1,1]\} & \{[3,4],[3,5]\} \\
\left\{\left[\frac{2}{7}, \frac{3}{4}\right],\left[\frac{1}{2}, \frac{2}{3}\right]\right\} & \{[1,3]\} & \{[1,1]\}
\end{array}\right), \\
\tilde{D}^{3}=\left(\begin{array}{ccc}
\{[1,1]\} & \left\{\left[\frac{1}{4}, \frac{2}{3}\right]\right\} & \{[2,4]\} \\
\{[1,2],[2,4]\} & \{[1,1]\} & \left\{\left[\frac{1}{3}, \frac{3}{5}\right]\right\} \\
\left\{\left[\frac{1}{3}, \frac{2}{5}\right]\right\} & \left\{\left[\frac{1}{6}, \frac{1}{3}\right],\left[\frac{1}{5}, \frac{1}{2}\right]\right\} & \{[1,1]\}
\end{array}\right) .
\end{gathered}
$$

In the following, we use the IMHFWA and IMHFWG operators to find the best university for the examinee.

1. Using IMHFWA operator based on algebraic operations:

- Step 1. First of all, adopt the IMHFWA operator to obtain the comprehensive value of university, $x_{i}$, expressed by three different decision makers:

$$
\begin{aligned}
& \tilde{\rho}_{1}^{1}=\{[0.4288,0.6441],[0.5183,0.7711]\}, \\
& \tilde{\rho}_{2}^{1}=\{[0.6869,1.1898],[0.9310,1.5962]\}, \\
& \tilde{\rho}_{3}^{1}=\{[0.8998,1.3208],[1.0328,1.5962]\}, \\
& \tilde{\rho}_{1}^{2}=\{[0.7100,1.1544],[0.6711,1.0801], \\
& \tilde{\rho}_{2}^{2}=\{[1.4101,1.9876],[1.4101,2.1748]\}, \\
& \tilde{\rho}_{3}^{2}=\{[0.7261,1.4101],[0.8171,1.3713]\}, \\
& \tilde{\rho}_{1}^{3}=\{[0.6894,1.5544]\}, \\
& \tilde{\rho}_{2}^{3}=\{[0.7472,1.1253],[1.0000,1.5198]\}, \\
& \tilde{\rho}_{3}^{3}=\{[0.4598,0.5513],[0.4736,0.6134]\} .
\end{aligned}
$$

- Step 2. Obtain the comprehensive performance value, $\tilde{\rho}_{i}$, for alternative $x_{i}$ based on IMHFWA operator (algebraic operations): 


$$
\begin{aligned}
& \tilde{\rho}_{1}=\{[0.6041,1.0838],[0.5918,1.0595], \\
& \text { [0.7325, 1.2506], [0.7193, 1.2244], } \\
& \text { [0.6369, 1.1361], [0.6244, 1.1112], } \\
& \text { [0.7680, 1.3071], [0.7545, 1.2803]\}, } \\
& \tilde{\rho}_{2}=\{[0.9223,1.4046],[1.0109,1.5451] \text {, } \\
& \text { [0.9223, 1.4538], [1.0109, 1.5971], } \\
& \text { [1.0109, 1.5451], [1.1035, 1.6937], } \\
& [1.0109,1.5971],[1.1035,1.7488]\} \text {, } \\
& \tilde{\rho}_{3}=\{[0.6854,1.0549],[0.6907,1.0820], \\
& \text { [0.7145, 1.0438], [0.7199, 1.0707], } \\
& \text { [0.7238, 1.1332], [0.7292, 1.1613], } \\
& \text { [0.7536, 1.1217], [0.7591, 1.1496]\}. }
\end{aligned}
$$

- Step 3. Using the score function, $s(\tilde{\rho})=$ $\sqrt[1 / \Delta g]{\prod_{\eta \in g} \eta^{+} \cdot \eta^{-}}$, defined in Section 2 , calculate the score of IMHFNs $\tilde{\rho}_{i}(i=1,2,3)$ :

$$
s\left(\tilde{\rho}_{1}\right)=0.7960, \quad s\left(\tilde{\rho}_{2}\right)=1.5850, \quad s\left(\tilde{\rho}_{3}\right)=0.7947 .
$$

Since $s\left(\tilde{\rho}_{2}\right)>s\left(\tilde{\rho}_{3}\right)>s\left(\tilde{\rho}_{1}\right)$, the most suitable university for the examinee is $x_{2}$.

2. Using IMHFWA operator based on Einstein operations:

- Step 1. Obtain the comprehensive value of university, $x_{i}$, expressed by three different decision makers:

$$
\begin{aligned}
& \tilde{\rho}_{1}^{1}=\{[0.4086,0.6340],[0.5041,0.7686]\} \\
& \tilde{\rho}_{2}^{1}=\{[0.6635,1.1736],[0.8795,1.5358]\} \\
& \tilde{\rho}_{3}^{1}=\{[0.8409,1.2171],[1.0000,1.5358]\} \\
& \tilde{\rho}_{1}^{2}=\{[0.6906,1.1355],[0.6447,1.0536], \\
& \tilde{\rho}_{2}^{2}=\{[1.0791,1.6272],[1.0183,1.5206]\} \\
& \tilde{\rho}_{3}^{2}=\{[0.7092,1.3722],[0.8104,1.3297]\} \\
& \tilde{\rho}_{1}^{3}=\{[0.6379,1.4895]\} \\
& \tilde{\rho}_{2}^{3}=\{[0.7331,1.1038],[0.9620,1.4509]\} \\
& \tilde{\rho}_{3}^{3}=\{[0.4410,0.5400],[0.4565,0.6052]\}
\end{aligned}
$$

- Step 2. Obtain the comprehensive performance value, $\tilde{\rho}_{i}$, for alternative $x_{i}$ based on IMHFWA operator (Einstein operations):

$$
\begin{aligned}
\tilde{\rho}_{1}=\{[0.5717,1.0453],[0.5577,1.0190], & {[0.6775,1.1868],[0.6622,1.1581], } \\
& {[0.6080,1.1042],[0.5936,1.0769], } \\
& {[0.7174,1.2510],[0.7016,1.2213]\}, } \\
\tilde{\rho}_{2}=\{[0.8901,1.3723],[0.9713,1.4987], & \\
& {[0.8901,1.4146],[0.9713,1.5438], } \\
& {[0.9710,1.4987],[1.0572,1.6336], } \\
& {[0.9713,1.5438],[1.0572,1.6817]\}, } \\
\tilde{\rho}_{3}=\{[0.6512,0.9953],[0.6575,1.0259], & \\
& {[0.6825,0.9839],[0.6889,1.0143], } \\
& {[0.6951,1.0826],[0.7016,1.1150], } \\
& {[0.7275,1.0706],[0.7342,1.1027]\} . }
\end{aligned}
$$

- Step 3. Using the score function, $s(\tilde{\rho})=$ $\sqrt[1 / \Delta g]{\prod_{\eta \in g} \eta^{+} \cdot \eta^{-}}$, defined in Section 2, calculate the score of IMHFNs $\tilde{\rho}_{i}(i=1,2,3)$ :

$$
s\left(\tilde{\rho}_{1}\right)=0.7160, \quad s\left(\tilde{\rho}_{2}\right)=1.4757, \quad s\left(\tilde{\rho}_{3}\right)=0.7248 .
$$

Since $s\left(\tilde{\rho}_{2}\right)>s\left(\tilde{\rho}_{3}\right)>s\left(\tilde{\rho}_{1}\right)$, the most suitable university for the examinee is $x_{2}$.

3. Using IMHFWG operator based on algebraic operations:

- Step 1. Obtain the comprehensive value of university,$x_{i}$, expressed by three different decision makers:

$$
\begin{aligned}
& \tilde{\rho}_{1}^{1}=\{[0.3204,0.5833],[0.4403,0.7571]\},\left.\tilde{\rho}_{2}^{1}=[0.5306,1.0954],[0.6170,1.2498]\right\}, \\
&\left.\tilde{\rho}_{3}^{1}=[0.5306,0.7571],[0.8405,1.2498]\right\}, \\
& \tilde{\rho}_{1}^{2}=\{[0.5833,1.0445],[0.4910,0.9259], \\
& \\
& \tilde{\rho}_{2}^{2}=\{[0.7293,1.2238],[0.6051,1.0741]\}, \\
& \tilde{\rho}_{3}^{2}=\{[0.6170,1.1915],[0.7755,1.1337]\}, \\
& \tilde{\rho}_{1}^{3}=\{[0.4481,1.1876]\},
\end{aligned}
$$




$$
\begin{aligned}
& \tilde{\rho}_{2}^{3}=\{[0.6580,1.0000],[0.7755,1.1337]\}, \\
& \tilde{\rho}_{3}^{3}=\{[0.3539,0.4910],[0.3796,0.5685]\} .
\end{aligned}
$$

- Step 2. Obtain the comprehensive performance value, $\tilde{\rho}_{i}$, for alternative $x_{i}$ based on IMHFWA operator (Einstein operations):

$$
\begin{array}{r}
\tilde{\rho}_{1}=\{[0.4335,0.8779],[0.4111,0.8454], \\
{[0.4628,0.9203],[0.4383,0.8855],} \\
{[0.4851,0.9707],[0.4591,0.9331],} \\
\quad[0.5190,1.0199],[0.4907,0.9794]\}, \\
\tilde{\rho}_{2}=\{[0.7281,1.2129],[0.7700,1.2692], \\
\quad[0.7281,1.2252],[0.7700,1.2824], \\
\quad[0.7700,1.2692],[0.8154,1.3297], \\
\quad[0.7700,1.2824],[0.8154,1.3439]\}, \\
\tilde{\rho}_{3}=\{[0.4832,0.7413],[0.4957,0.7842], \\
\quad[0.5170,0.7315],[0.5306,0.7736], \\
{[0.5555,0.8633],[0.5706,0.9171],} \\
[0.5964,0.8511],[0.6130,0.9038]\} .
\end{array}
$$

- Step 3. Using the score function, $s(\tilde{\rho})=$ $\sqrt[1 / \Delta g]{\prod_{\eta \in g} \eta^{+} \cdot \eta^{-}}$defined in Section 2, calculate the score of IMHFNs $\tilde{\rho}_{i}(i=1,2,3)$ :

$$
s\left(\tilde{\rho}_{1}\right)=0.4278, \quad s\left(\tilde{\rho}_{2}\right)=0.9830, \quad s\left(\tilde{\rho}_{3}\right)=0.4445 .
$$

Since $s\left(\tilde{\rho}_{2}\right)>s\left(\tilde{\rho}_{3}\right)>s\left(\tilde{\rho}_{1}\right)$, the most suitable university for the examinee is $x_{2}$.

4. Using IMHFWG operator based on Einstein operations:

- Step 1. Obtain the comprehensive value of university, $x_{i}$, expressed by three different decision makers:

$$
\begin{aligned}
& \tilde{\rho}_{1}^{1}=\{[0.3304,0.5918],[0.4493,0.7592]\}, \\
& \tilde{\rho}_{2}^{1}=\{[0.5516,1.1099],[0.6576,1.2938]\}, \\
& \tilde{\rho}_{3}^{1}=\{[0.5745,0.8216],[0.8688,1.2938]\}, \\
& \tilde{\rho}_{1}^{2}=\{[0.6012,1.0613],[0.5140,0.9491], \\
& \tilde{\rho}_{2}^{2}=\{[1.2219,1.7433],[1.2219,1.8208]\}, \\
& \tilde{\rho}_{3}^{2}=\{[0.6329,1.2219],[0.7822,1.1666]\},
\end{aligned}
$$

$$
\begin{aligned}
& \tilde{\rho}_{1}^{3}=\{[0.4715,1.2340]\}, \\
& \tilde{\rho}_{2}^{3}=\{[0.6714,1.0191],[0.8077,1.1823]\}, \\
& \tilde{\rho}_{3}^{3}=\{[0.3645,0.4983],[0.3894,0.5745]\} .
\end{aligned}
$$

- Step 2. Obtain the comprehensive performance value, $\tilde{\rho}_{i}$, for alternative $x_{i}$ based on IMHFWA operator (Einstein operations):

$$
\begin{aligned}
\tilde{\rho}_{1}= & \{[0.4516,0.9046],[0.4297,0.8727], \\
& {[0.4896,0.9587],[0.4654,0.9243], } \\
& {[0.5023,0.9914],[0.4774,0.9555], } \\
& {[0.5454,1.0525],[0.5180,1.0137]\}, } \\
\tilde{\rho}_{2}= & \{[0.7554,1.2406],[0.8042,1.3072], \\
& {[0.7554,1.2561],[0.8042,1.3239], } \\
& {[0.8042,1.3072],[0.8571,1.3789], } \\
& {[0.8042,1.3239],[0.8571,1.3969]\}, } \\
\tilde{\rho}_{3}= & \{[0.5069,0.7790],[0.5189,0.8209], \\
& {[0.5415,0.7686],[0.5544,0.8098], } \\
& {[0.5769,0.8974],[0.5909,0.9478], } \\
& {[0.6174,0.8850],[0.6326,0.9344]\} . }
\end{aligned}
$$

- Step 3. Using the score function, $s(\tilde{\rho})=$ $\sqrt[1 / \Delta g]{\prod_{\eta \in g} \eta^{+} \cdot \eta^{-}}$defined in Section 2, calculate the score of IMHFNs $\tilde{\rho}_{i}(i=1,2,3)$ :

$$
s\left(\tilde{\rho}_{1}\right)=0.4632, \quad s\left(\tilde{\rho}_{2}\right)=1.0585, \quad s\left(\tilde{\rho}_{3}\right)=0.4826 .
$$

Since $s\left(\tilde{\rho}_{2}\right)>s\left(\tilde{\rho}_{3}\right)>s\left(\tilde{\rho}_{1}\right)$, the most suitable university for the examinee is $x_{2}$.

During the above analysis, we used IMHFWA operator (Algebraic operations), IMHFWA operator (Einstein operations), IMHFWG operator (Algebraic operations), and IMHFWG operator (Einstein operations) to deal with the voluntary choice of college entrance examination problem in China. The results show that the final choice for the examinee is always the second university. However, the scores of the aggregated IMHFNs based on four different aggregation operators are different. For different operations, the scores based on IMHFWA operator are always bigger than those based on IMHFWG operator. On the other hand, for IMHFWA operator, the aggregated results based on algebraic operations are bigger than those based 
on Einstein operations. For IMHFWG operator, the aggregated results based on algebraic operations are smaller than those based on Einstein operations.

\section{Conclusions and future works}

In this paper, we introduced the IMHFS, which is properly complementary to the existing HFS theory. The main contributions of this paper include the following three aspects. First of all, we have extended the multiplicative intuitionistic fuzzy preference relations [12] to a more generalized form and proposed the IMHFS, which is a powerful technique to describe the preference information assessed by different appraise subjective and is very useful in decision-making problems. Then, we have proposed a series of aggregation operators for aggregating interval-valued multiplicative hesitant fuzzy information, including IMHFWA, IMHFWG, IMHFOWA, and IMHFOWG operators, based on which a new multi-criteria GDM method is proposed. Finally, we have investigated the problem of voluntary choice of college entrance examination in China based on the theory proposed in this paper.

The future study mainly focuses on the following two aspects:

1. Strengthening the research of aggregation operators for IMHFNs, e.g. considering the relationship between the data to be aggregated;

2. Applying the multi-criteria GDM method proposed in this paper to many other areas such as supply chain management, personnel selection, pattern recognition, and data mining.

\section{Acknowledgment}

The authors would like to thank the Editor-in-Chief and anonymous reviewers. This work has been supported by China National Natural Science Foundation (No. 71301142), Zhejiang Science \& Technology Plan of China (2015C33024), and Zhejiang Provincial Natural Science Foundation of China (No. LQ13G010004) and funded by China Postdoctoral Science Foundation (No. 2014M550353) and the National Education Information Technology Research (No. 146242069).

\section{References}

1. Xu, Z.S. "Priority weight intervals derived from intuitionistic multiplicative preference relations", IEEE Transactions on Fuzzy Systems, 21(4), pp. 642-654 (2013a).

2. Li, D.F. and Wan, S.P. "Fuzzy heterogeneous multiattribute decision making method for outsourcing provider selection", Expert Systems with Applications, 41(6), pp. 3047-3059 (2014a).
3. Wang, L., Ni, M., Yu, Z. and Zhu, L. "Power geometric operators of hesitant multiplicative fuzzy numbers and their application to multiple attribute group decision making", Mathematical Problems in Engineering, Article ID 186502, 16 pages (2014).

4. Xu, Z.S. "Intuitionistic preference relations", In Intuitionistic Preference Modeling and Interactive Decision Making, pp. 1-193, Springer Berlin Heidelberg (2014).

5. Chiclana, F., Herrera, F. and Herrera-Viedma, E. "Integrating three representation models in fuzzy multipurpose decision making based on fuzzy preference relations", Fuzzy Sets and Systems, 97(1), pp. 33-48 (1998).

6. Li, D.F. and Wan, S.P. "A fuzzy inhomogeneous multiattribute group decision making approach to solve outsourcing provider selection problems", KnowledgeBased Systems, 67, pp. 71-89 (2014b).

7. Chiclana, F., Herrera, F. and Herrera-Viedma, E. "Integrating multiplicative preference relations in a multipurpose decision-making model based on fuzzy preference relations", Fuzzy Sets and Systems, 122(2), pp. 277-291 (2001).

8. Yager, R.R. "OWA aggregation over a continuous interval argument with applications to decision making", IEEE Transactions on Systems, Man, and Cybernetics, Part B: Cybernetics, 34(5), pp. 1952-1963 (2004).

9. Xu, Z.S. "AC-OWA operator-based approach to decision making with interval fuzzy preference relation", International Journal of Intelligent Systems, 21(12), pp. 1289-1298 (2006).

10. Fan, Z.P., Ma, J., Jiang, Y.P., Sun, Y.H. and Ma, L. "A goal programming approach to group decision making based on multiplicative preference relations and fuzzy preference relations", European Journal of Operational Research, 174(1), pp. 311-321 (2006).

11. Genç, S., Boran, F.E., Akay, D. and Xu, Z. "Interval multiplicative transitivity for consistency, missing values and priority weights of interval fuzzy preference relations", Information Sciences, $\mathbf{1 8 0}(24)$, pp. 48774891 (2010).

12. Xia, M.M., Xu, Z.S. and Liao, H.C. "Preference relations based on intuitionistic multiplicative information", IEEE Transactions on Fuzzy Systems, 21(1), pp. 113-133 (2013).

13. Jiang, Y., Xu, Z. and Yu, X. "Compatibility measures and consensus models for group decision making with intuitionistic multiplicative preference relations", Applied Soft Computing, 13(4), pp. 2075-2086 (2013).

14. Xia, M.M. and Xu, Z.S. "Group decision making based on intuitionistic multiplicative aggregation operators", Applied Mathematical Modelling, 37(7), pp. 5120-5133 (2013a).

15. Jiang, Y. and Xu, Z. "Aggregating information and ranking alternatives in decision making with intuitionistic multiplicative preference relations", Applied Soft Computing, 22, pp. 162-177 (2014). 
16. Yu, D.J. and Fang, L. "Intuitionistic multiplicative aggregation operators with their application in group decision making", Journal of Intelligent and Fuzzy Systems, 27(1), pp. 131-142 (2014).

17. Yu, S. and Xu, Z.S. "Aggregation and decision making using intuitionistic multiplicative triangular fuzzy information", Journal of Systems Science and Systems Engineering, 23(1), pp. 20-38 (2014).

18. Xia, M.M. "Research on fuzzy decision information aggregation techniques and measures", Doctoral dissertation, Southeast University (2012).

19. Torra, V. "Hesitant fuzzy sets", International Journal of Intelligent Systems, 25(6), pp. 529-539 (2010).

20. Rodríguez, R.M., Martínez, L., Torra, V., Xu, Z.S. and Herrera, F. "Hesitant fuzzy sets: state of the art and future directions", International Journal of Intelligent Systems, 29(6), pp. 495-524 (2014).

21. Bedregal, B., Reiser, R., Bustince, H., Lopez-Molina, C. and Torra, V. "Aggregation functions for typical hesitant fuzzy elements and the action of automorphisms", Information Sciences, 255, pp. 82-99 (2014).

22. Xia, M.M. and Xu, Z.S. "Managing hesitant information in GDM problems under fuzzy and multiplicative preference relations", International Journal of Uncertainty, Fuzziness and Knowledge-Based Systems, 21(6), pp. 865-897 (2013b).

23. Yu, D.J. "Group decision making under multiplicative hesitant fuzzy environment", International Journal of Fuzzy Systems, 16(2), pp. 233-241 (2014).

24. Wang, J.Q., Zhou, P., Li, K.J., Zhang, H.Y. and Chen, X.H. "Multi-criteria decision-making method based on normal intuitionistic fuzzy-induced generalized aggregation operator", TOP, 22(3), pp. 1103-1122 (2014).

25. Liao, H.C., Xu, Z.S. and Xia, M.M. "Multiplicative consistency of hesitant fuzzy preference relation and its application in group decision making", International Journal of Information Technology \& Decision Making, 13(1), pp. 47-76 (2014).

26. Yu, D.J., Merigó, J.M. and Zhou, L.G. "Intervalvalued multiplicative intuitionistic fuzzy preference relations", International Journal of Fuzzy Systems, 15(4), pp. 412-422 (2013).

27. Merigó, J.M., Casanovas, M. and Yang, J.B. "Group decision making with expertons and uncertain generalized probabilistic weighted aggregation operators",
European Journal of Operational Research, 235(1), pp. 215-224 (2014).

28. Xu, Z.S. "Group decision making model and approach based on interval preference orderings", Computers \& Industrial Engineering, 64(3), pp. 797-803 (2013b).

29. Xu, Z.S. and Cai, X.Q. "On consensus of group decision making with interval utility values and interval preference orderings", Group Decision and Negotiation, 22(6), pp. 997-1019 (2013).

\section{Biographies}

De-jian Yu received the BS degree in Information Management and Information System from Heilongjiang Institute of Science and Technology, Harbin, China, the MS degree in System Engineering from Nanjing University of Aeronautics and Astronautics, Nanjing, China, and the $\mathrm{PhD}$ degree in Management Science and Engineering from Southeast University, Nanjing, China, in 2012. He is currently a lecturer at the School of Information, Zhejiang University of Finance and Economics, Hangzhou, China. He has authored or coauthored more than 30 journal papers. His current research interests include aggregation operators, information fusion, and multi-criteria decision making.

Deng-Feng Li was born in 1965 . He received the BSc and MSc degrees in Applied Mathematics from the National University of Defense Technology, Changsha, China, in 1987 and 1990, respectively, and the PhD degree in System Science and Optimization from the Dalian University of Technology, Dalian, China, in 1995. From 2003 to 2004, he was a visiting scholar at the School of Management, University of Manchester Institute of Science and Technology, Manchester, UK. He is currently a professor at the School of Management, Fuzhou University, Fuzhou, China. He has authored or coauthored more than 200 journal papers and four monographs. His current research interests include fuzzy decision analysis, group decision making, fuzzy game theory, supply chain, fuzzy sets and system analysis, fuzzy optimization, and differential game. 